\title{
Les techniques du chant villageois dans les Alpes dinariques (Yougoslavie)
}

\section{Ankica Petrović}

Traducteur : Isabelle Schulte-Tenckhoff

\section{OpenEdition \\ Journals}

Édition électronique

URL : http://journals.openedition.org/ethnomusicologie/1578

ISSN : 2235-7688

Éditeur

ADEM - Ateliers d'ethnomusicologie

\section{Édition imprimée}

Date de publication : 1 octobre 1991

Pagination : 103-115

ISBN : 978-2-8257-0431-8

ISSN : 1662-372X

Référence électronique

Ankica Petrović, «Les techniques du chant villageois dans les Alpes dinariques (Yougoslavie) »,

Cahiers d'ethnomusicologie [En ligne], 4 | 1991, mis en ligne le 01 janvier 2012, consulté le 01 mai 2019. URL : http://journals.openedition.org/ethnomusicologie/1578 


\title{
LES TECHNIQUES DU CHANT VILLAGEOIS DANS LES ALPES DINARIQUES (YOUGOSLAVIE)*
}

\author{
Ankica Petrović
}

Tenter d'expliquer scientifiquement les techniques vocales au sein d'une culture, puis d'une période à l'intérieur de celle-ci est une tâche complexe. Si la théorie ethnomusicologique n'offre guère de points de repère à ce sujet, pas plus que les études monographiques d'ailleurs, cela ne veut pas dire pour autant que cet aspect de la pratique musicale soit dépourvu de signification. $\mathrm{Au}$ contraire, l'étude des techniques vocales et de leur application à des répertoires, des formes et des fonctions culturellement déterminés contribue largement à notre connaissance des styles musicaux et de leurs composantes.

Le manque d'intérêt que les chercheurs ont manifesté à l'égard des techniques vocales et de leur rôle dans la formation du style musical s'explique peutêtre par la rareté des transcriptions, due à l'absence de signes correspondants dans notre système conventionnel de notation qui ne valorise qu'une seule forme culturelle et se borne à en restituter la pratique musicale, prétendument supérieure. Dans les limites de cette pratique même, seuls certains caractères stylistiques ont été relevés: rythme, hauteur, mélodie, interdépendance polyphonique et dynamique. En revanche, d'autres éléments techniques qui ne contribuent pas moins au style, sont considérés comme allant de soi. Ainsi, la technique du bel canto est à tel point répandue chez nous qu'on ne se donne pas la peine de l'indiquer à l'aide de signes spécifiques. Si l'exécution du bel canto n'atteint son expressivité caractéristique qu'au moyen d'une technique vocale appropriée, on ne peut pas toujours être sûr que les modes d'exécution actuels correspondent réellement, du point de vue technique, à ceux pratiqués au temps de Bellini et de Donizetti. La même incertitude règne au sujet de l'authenticité des techniques vocales comme à celui des styles instrumentaux de la musique classique européenne. Par exemple, les interprétations contemporaines des sonates de Beethoven se rapprochent-elles de l'«idéal» que représente l'interprétation originelle à l'époque du compositeur?

Le problème se complique lorsqu'on se tourne vers les musiques non occidentales dont les techniques et les styles sont déterminés exclusivement par la tradition locale, reflétant ainsi la pérennité d'un environnement socioculturel replié sur lui-même.

Bien que la voix humaine apparaisse comme le produit sonore unique d'un organe vocal partout identique, chaque culture lui fait subir un traitement

* Traduit de l'anglais par Isabelle Schulte-Tenckhoff. 
technique particulier et l'exerce de diverses façons. Il en résulte un éventail d'éléments stylistiques et musicaux.

Les Alpes dinariques en Yougoslavie centrale se distinguent par des techniques vocales et donc des caractères musicaux spécifiques. C'est une région habitée par des Serbes, des Croates et des Musulmans. Bien que ses zones limitrophes en Yougoslavie orientale, en Albanie et dans la région du Rhodope en Bulgarie possèdent traditionnellement des techniques vocales et des phénomènes musicaux semblables, nous nous limiterons, dans cet article, aux montagnes dinariques elles-mêmes, car elles abritent encore, à notre avis, les traits et les techniques les plus rudimentaires de la musique des anciens Balkans.

Les Alpes dinariques sont pour la plupart formées de chaînes calcaires, mais elles n'en constituent pas moins deux zones distinctes en vertu de la végétation et du climat: la zone méditerranéenne, proche de l'Adriatique, et la zone continentale. L'élevage y joue depuis toujours le rôle économique principal. Difficiles d'accès pour des raisons topographiques, les montagnes dinariques sont jusqu'à ce jour restées partiellement isolées des influences externes. Elles ont pourtant toujours été habitées par de de petites communautés d'éleveurs.

La vie de ces montagnards se déroulant principalement en plein air, toute activité - qu'elle soit de production ou de création - est adaptée aux conditions naturelles. Le chant est surtout considéré comme un moyen de communication, encore qu'il soit placé à un niveau artistique plus élevé. La plupart des genres vocaux sont de nature collective, car on les exécute avant tout en groupe et en public, tant pour cultiver l'art du chant que pour en faire bénéficier les auditeurs. Seuls certains types de chant relèvent du répertoire intimiste, telles les berceuses et chansons en solo des bergers, qui exigent des techniques vocales spécifiques et dont l'exécution intervient nettement moins souvent au cours de la vie ordinaire que celle des chansons collectives. La même remarque s'applique à la musique instrumentale. Traditionnellement, dans la région considérée, les instruments se limitent le plus souvent aux aérophones, comme le diple ${ }^{1}$ et la flûte à un ou deux tuyaux ${ }^{2}$. On en joue individuellement, en improvisant pour son propre plaisir. Le caractère de cette musique implique le plus souvent un faible volume sonore, auquel la technique de jeu est adaptée. A côté des aérophones mentionnés, on joue aussi de la vièle monocorde gusle ${ }^{3}$ qui sert à accompagner les récitatifs, généralement des chants épiques. Dans ce cas, la technique vocale et instrumentale est adaptée aux exigences du genre:

Le diple est un ancien instrument pastoral, soit une clarinette à deux tuyaux - d'où son nom (d'après le grec diploós, «double») - faits de deux roseaux individuels. On y ajoute le plus souvent une peau de chèvre en guise de réservoir d'air, ce qui en fait une cornemuse. On s'en sert principalement pour improviser des airs pastoraux.

2 Les expressions jednocjevne y dvocjevne svirale, ou jednojke et dvojnice signifient flûte à un ou deux tuyaux. Traditionnellement destinées à l'improvisation, elles sont utilisées plus récemment pour accompagner les danses populaires (kola).

3 Le gusle est une vièle monocorde exclusivement utilisée pour accompagner les contes chantés par les hommes, généralement des épopées. 


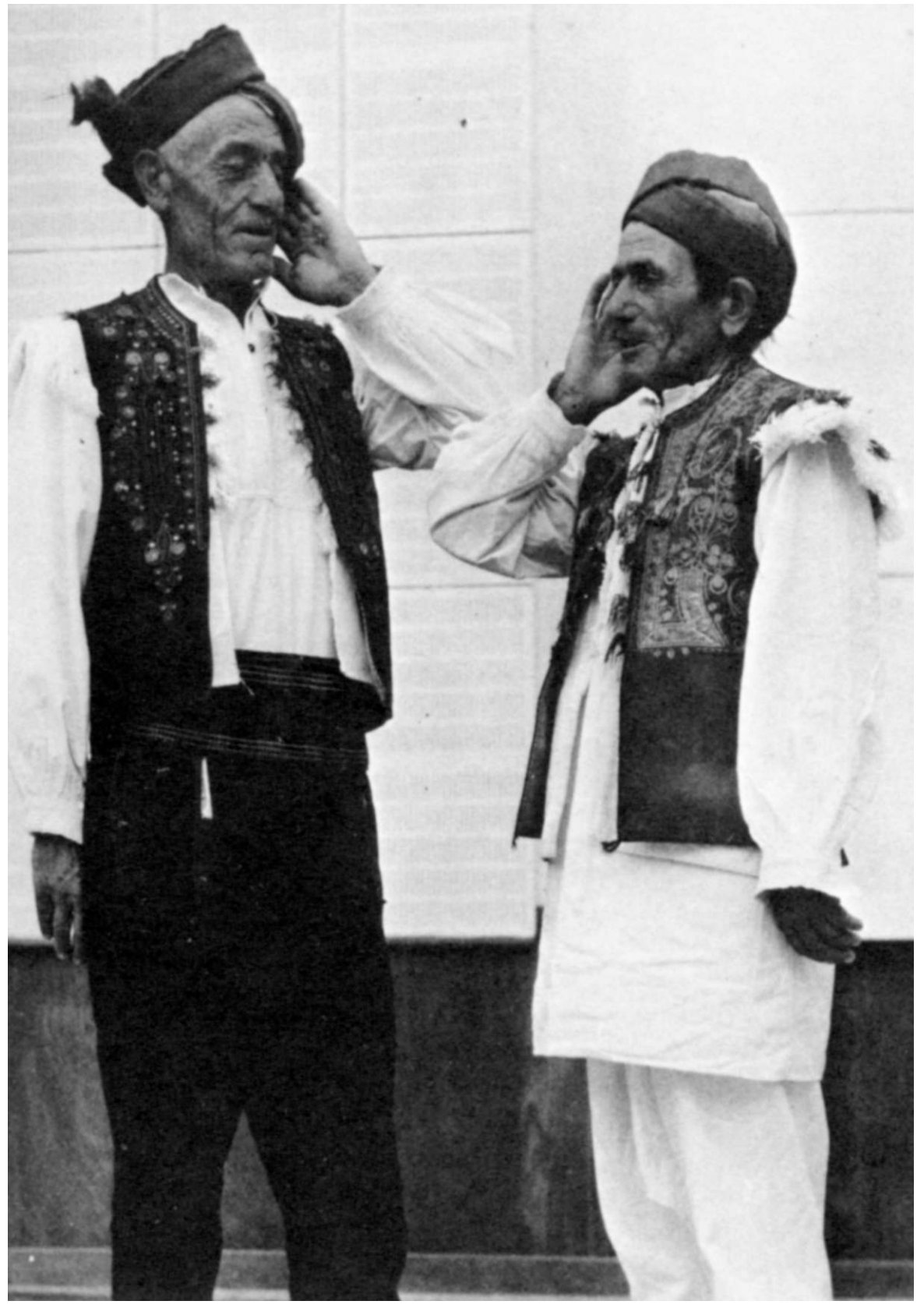

Fig. 1: Interprétation d'un kiridžijske pjesme, Bosnie occidentale. 
les interprètes aspirent à atteindre le meilleur équilibre entre le chant et le jeu du gusle, du point de vue tant stylistique que technique.

Mais, comme nous l'avons déjà dit, l'aspect communicatif peut revêtir autant d'importance que l'aspect artistique. Dans ce cas, on veille à une émission forte pour que le chant puisse être entendu sur une distance aussi grande que possible. Or, ce n'est guère là une question de communication verbale au sens littéral du terme. La prononciation du texte est, au contraire, souvent si inarticulée que le contenu en devient inintelligible, en dépit de l'adhésion aux normes poétiques locales. Ici, la composante sonore acquiert donc une signification symbolique accrue, et il n'est pas exagéré d'affirmer qu'on a affaire à un son artistiquement créé, doté d'une fonction symbolique et communicative précise, où chaque élément stylistique et technique possède une valeur et un sens propres. D'une manière générale, de telles chansons expriment l'appartenance régionale, l'esprit de fête, le chagrin (dans le cas des chants funèbres), la solitude, l'amour passionnel ou l'appel de l'être bien-aimé. Il n'est cependant pas nécessaire que le texte de la chanson corresponde aux états d'âme suscités par la musique.

Chaque catégorie traditionnelle du chant populaire des montagnes dinariques présente ses propres traits stylistiques permettant de l'identifier sur tout le territoire. D'autre part, des traits régionaux plus spécifiques encore apparaissent dans certains symboles sonores traduisant l'origine du chanteur et de son public virtuel. Le plus souvent, l'exécution ou l'audition des chansons se fait sur une certaine distance, et seuls les auditeurs de même origine comprennent sans autre la signification des symboles sonores employés.

Celui qui étudie ces chansons est obligatoirement renvoyé à une approche de type émique. C'est en effet la seule méthode qui permette de distinguer, dans le cadre d'une culture donnée, les éléments stylistiques ayant valeur générique de ceux à valeur particulière, puis de déterminer le mode de fonctionnement de ces éléments selon les régions et les villages plus restreints, et selon le sexe et le groupe d'âge. Ces deux derniers critères - y compris les specificités physiologiques de l'expression vocale, que des techniques particulières sont susceptibles de renforcer - permettent notamment de déterminer le statut des chanteurs par opposition à ses destinataires.

Pour les membres d'une culture donnée, de tels repères sont essentiels, car avec le temps ils ont cristallisé des symboles musicaux inhérents à un contexte socioculturel seul capable de les rendre intelligibles. Ainsi dans la région considérée, les défenseurs de la tradition rurale qui la caractérise acceptent les critères tant génériques que particuliers du chant authentique. En revanche, dans le contexte urbain, même géographiquement proche, les qualités artistiques de cette musique sont niées. Pour les «gens d'ailleurs", dotés ou non d'une formation musicale, ce sont des sons discordants, laids, voire même criards ou braillards sans valeur et, de ce fait, impropres à être considérés comme des créations musicales.

Quant aux villageois des Alpes dinariques, ils continuent à valoriser leur musique traditionnelle qu'ils opposent favorablement à tous les autres genres 
musicaux auxquels ils ont maintenant accès grâce aux médias: ils n'en apprécient aucun, qu'il s'agisse de la musique classique ou populaire européenne ou de la musique populaire urbaine de Yougoslavie. Mais c'est la musique classique européenne qu'ils rejettent le plus: tous ses genres - vocal, instrumental, ou les deux à la fois - tombent sous un seul dénominateur, l' "opéra ». Lorsque nous avons fait écouter un enregistrement sur bande magnétique de l'aria de Cho-Cho-San de «Madame Butterfly» de Puccini à un groupe de jeunes filles d'un village de la région de Visočica (près de Sarajevo), celles-ci ont tourné en ridicule l'interprétation vocale. D'après elles, la mélodie n'était même pas belle, et l'interprète la «beuglait» (c'est pourtant le piano qui domine l'aria en question). Il est intéressant de noter que ces mêmes jeunes filles pratiquent une forme de chant à intensité dynamique exagérée, qu'elles considèrent pourtant comme une manière de chanter fort cultivée. En fait, le registre aigu de l'aria en question, inconnu dans leur pratique vocale, leur répugne, parce qu'elles le jugent «braillard».

A première vue, il semblerait que la tradition musicale villageoise de Yougoslavie centrale ignore certains systèmes de composition et principes techniques du chant. D'autre part, les villageois semblent incapables d'expliquer les caractères de leur tradition musicale. Or, lorsqu'on se penche sur celle-ci, on perçoit un niveau élevé de conscience collective quant aux structures musicales sous-tendant chaque genre - le terme de «conscience collective» se référant ici au fait que les interprètes, tout comme leur audience, ont une idée très claire des traits essentiels de leurs genres musicaux. La pratique démontre que même ceux qui ne font qu'écouter la musique parviennent à en expliquer, comme les chanteurs eux-mêmes, les éléments stylistiques, puis à mettre en évidence les «bonnes » attitudes esthétiques face aux genres musicaux. En d'autres termes, ils les apprennent et les vivent d'une manière similaire aux interprètes. Mais lorsqu'il est question de technique vocale, les auditeurs ne parviennent à l'expliquer que superficiellement, les explications techniques plus détaillées et complexes revenant à ceux qui ont réellement une expérience vocale et sont, de ce fait, en mesure de préciser certains processus techniques du point de vue physiologique.

Les principaux traits des techniques vocales considérées ici seront expliqués à l'aide d'exemples provenant des deux catégories de chant les plus répandues: les potresalice, chansons vibrantes, et les ganga.

La première catégorie, considérée comme la plus ancienne, fait apparaître une grande diversité de fonctions et de contenus qui donne lieu, à son tour, à une variété similaire dans l'organisation et l'exécution musicales. Les types suivants de chansons tombent sous cette catégorie:

- chansons vibrantes de voyageurs et chansons de marchands ambulants, appelées kiridžijske pjesme ${ }^{4}$;

$4 \quad$ Les kiridžijske pjesme sont les chansons des marchands ambulants, interprétées comme chansons vibrantes à une ou deux voix. 


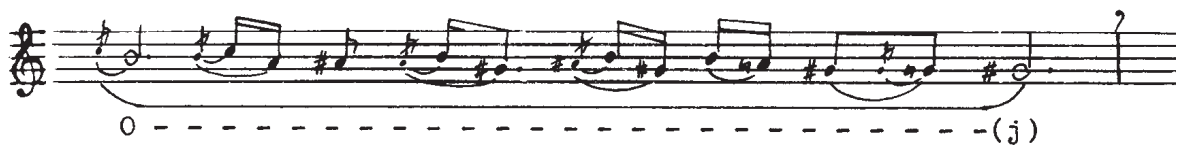

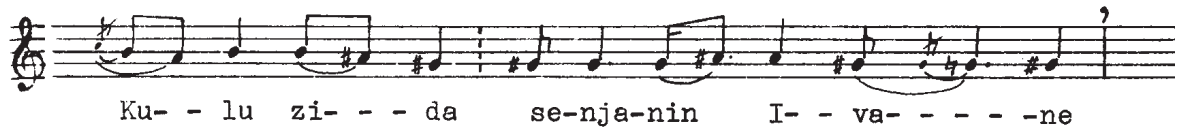

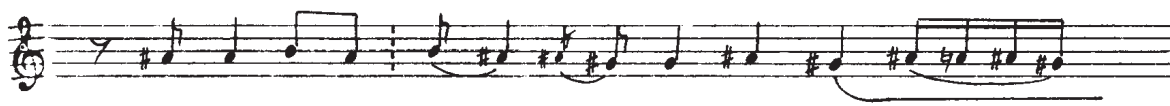
8 u vi-si-nu $\mathrm{za}$ de-vet ta- -va-

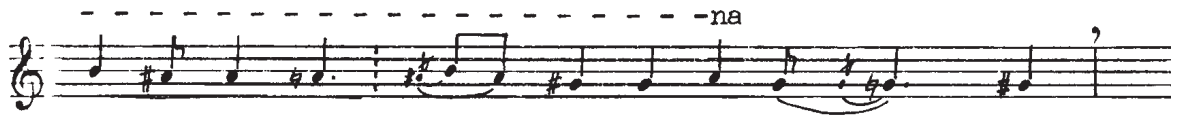
u ši-ri- - nu za de- set o- da- - - - ja

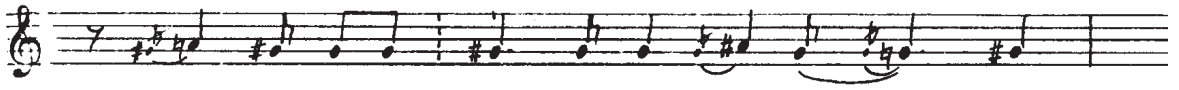
to se ču-do na da-le- - ko ču- - . - -10

t) za to ču-o ca- - -re su- -lej- -ma- ......

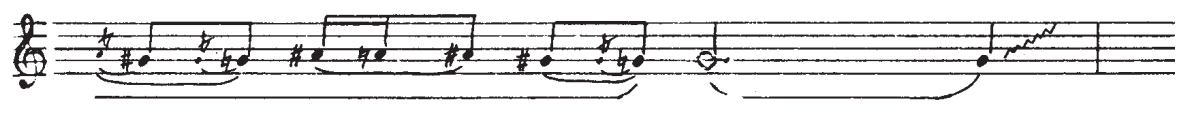

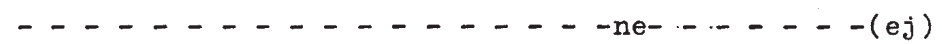

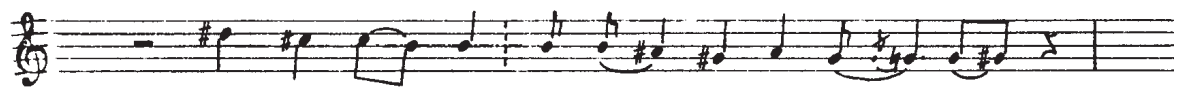
knjigu pi- - še $i$ na nju se smi--je

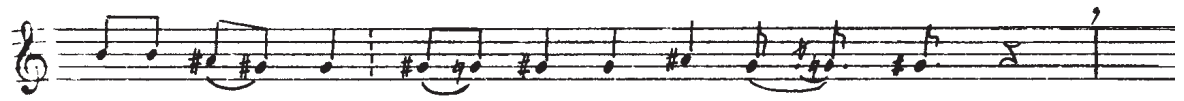
pa je pi- -še se- - -nja-nin I- -va- - - -nu

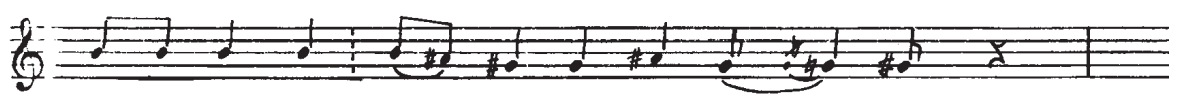
oj I- -va- -ne sinj- -ski gos-po- -da- - - re

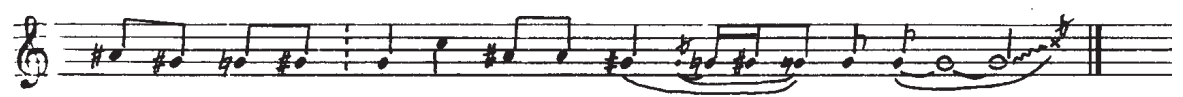
ču-o je-sam a vi-di-o ni- ...- sam oj

Fig. 2: Chanson épique vibrante. 


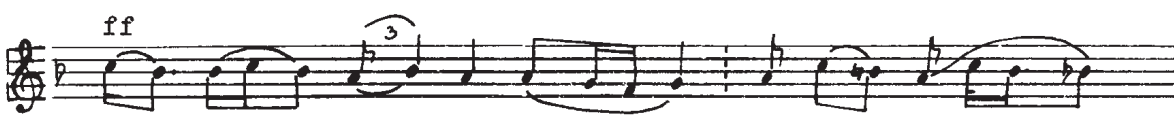

$0 j$ pjev- ni_ pjevče___ spremaj se

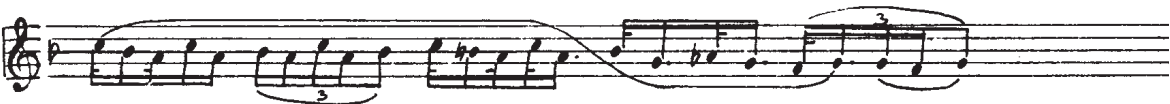
dje- - - - - - - f $_{1}-\ldots-\cdots$

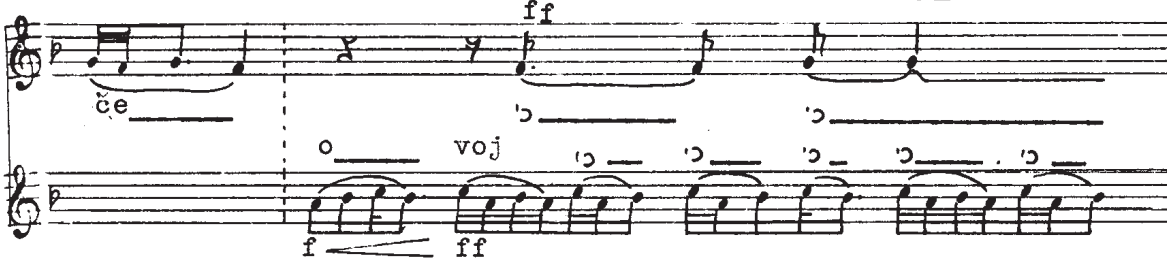

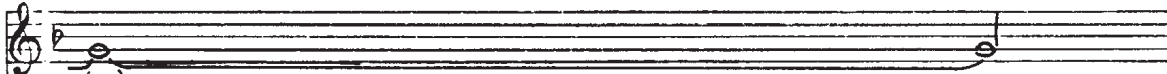
(כ)

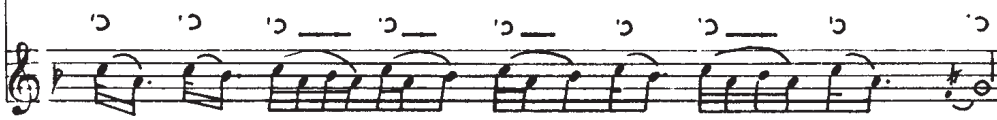
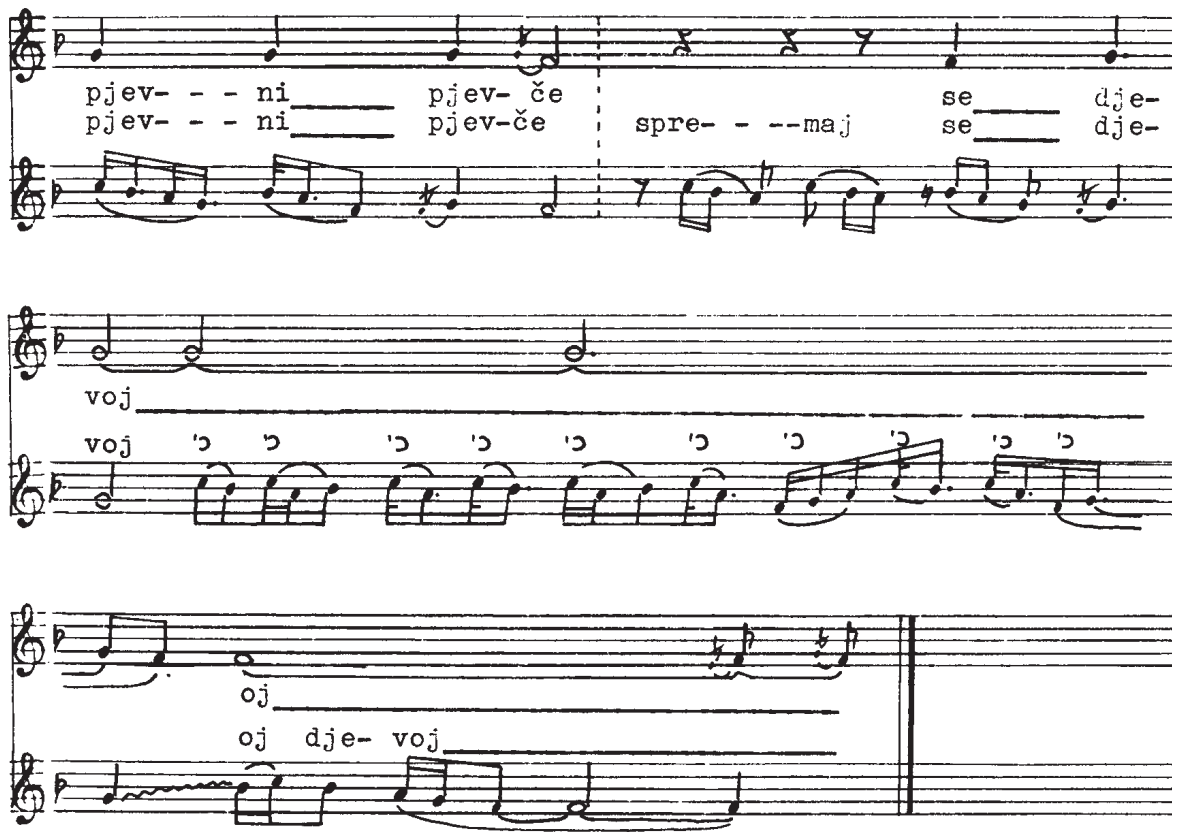

Fig. 3: Chanson vibrante de mariage. 

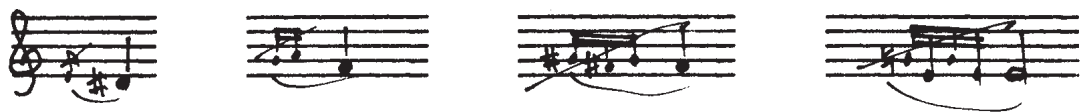

Fig. 4: Les méthodes du «sanglot» dans la ganga.

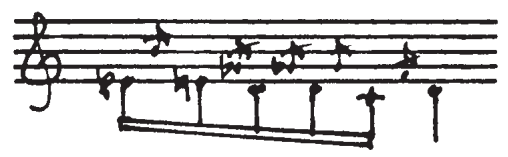

Fig. 5: Présentation du «sanglot» takiyah, selon la transcription de Caton.

- chansons vibrantes de mariage et turčije ${ }^{5}$;

- chansons vibrantes à boire, c'est-à-dire destinées à porter des toasts ;

- chansons vibrantes sur textes provenant des chants épiques;

- chansons vibrantes sur textes provenant des chansons de berger.

Toutes ces formes de chansons vibrantes sont représentées dans la région plus large de Bosnie-Herzégovine, ainsi que sur le territoire de Lika en Croatie, où on les appelle rozgalice. Une tradition de chant similaire existe chez les Albanais Malisos du Monténégro oriental et en Albanie voisine, où on la désigne par le terme de «chant de l'oreille». Cette dénomination albanaise est attribuée à la pratique de l'interprète de mettre sa main en cornet derrière l'oreille pendant le chant, comme on le fait d'ailleurs également dans toutes les autres régions mentionnées (Munishi 1987).

La plupart des chansons de la première catégorie sont pratiquées par les hommes, que ce soit en solo, en duo ou en petit ensemble. Mais en Bosnie occidentale, les chansons à boire et les chansons vibrantes pastorales peuvent aussi être exécutées par les femmes âgées, dont le statut est l'équivalent de celui des hommes.

La caractéristique principale de toutes les chansons vibrantes est une distribution unique qui fait se succéder le récitatif (parfois précédé d'une brève exclamation) et l'exclamation. Le premier se distingue par une émission syllabique, la seconde par une émission hautement mélismatique. Dans ce dernier cas, l'ornementation revient en général à un seul chanteur. Cette méthode vocale a été relevée et décrite en détail par Alberto Fortis, voyageur et encyclopédiste italien qui visita la Dalmatie et son arrière-pays en 1774. Les observations de Fortis (voir 1984) ont également porté sur les habitants des montagnes dinariques:

Le terme de turčije désigne les chansons vibrantes pratiquées dans l'Herzégovine orientale, à la manière turque. 

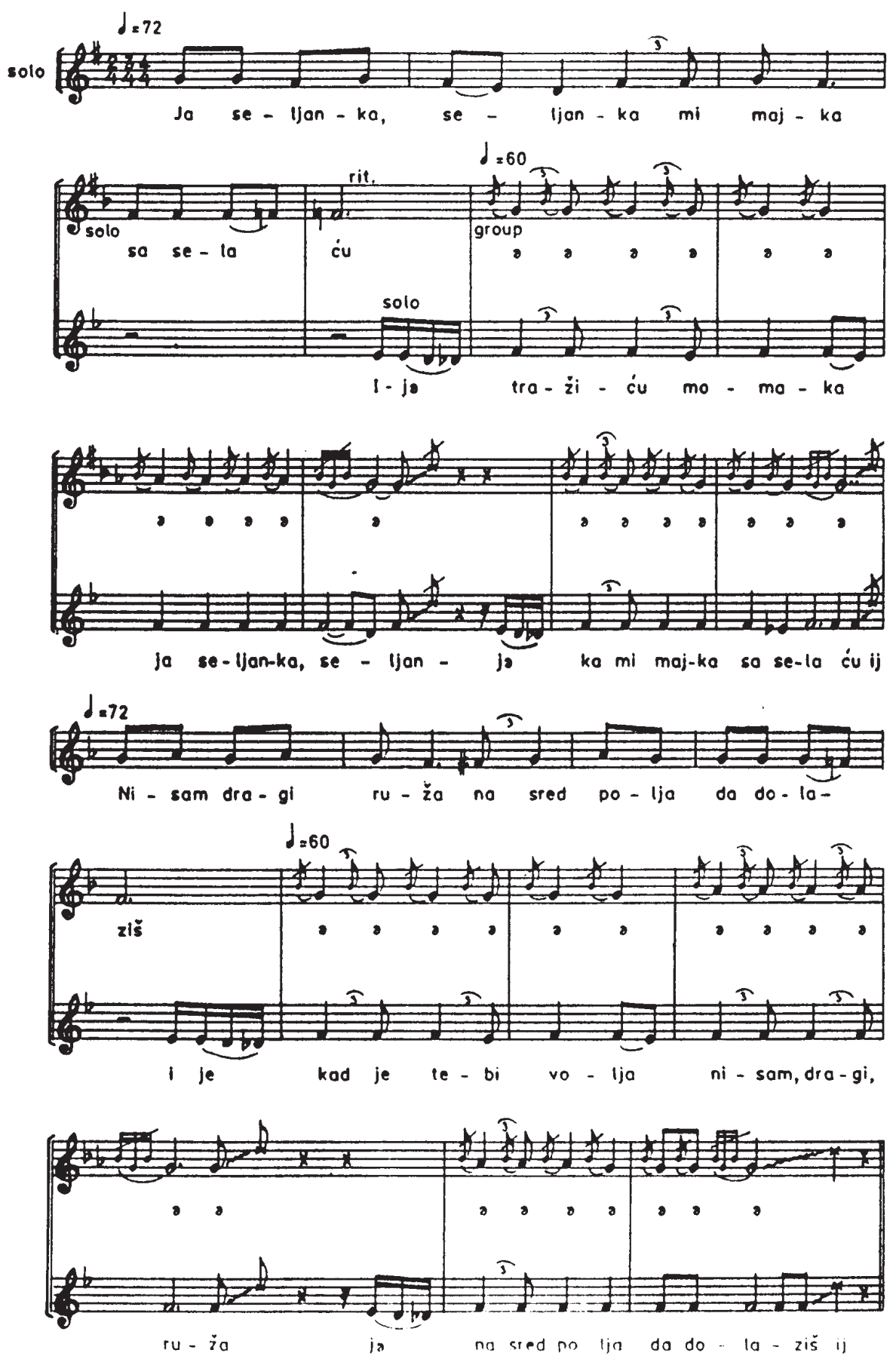

Fig. 6: Transcription d'une ganga de Herzégovine. 
En traversant les montagnes désertes, surtout la nuit, le Morlak ${ }^{6}$ chante les hauts faits des nobles et des rois slaves ou bien quelque tragédie. Si par chance un autre voyageur longe le flanc d'une montagne voisine, il répète les vers chantés par le premier, et cet échange continue jusqu'à ce que les deux voix soient séparées par la distance. Chaque vers est précédé d'une longue exclamation, un «ô» aux résonances barbares; les paroles sont débitées rapidement, sans aucune inflexion vocale qui, elle, est réservée à la dernière syllabe; le vers est conclu par une autre longue exclamation exécutée avec un vibrato renforcé par l'expiration. (Fortis 1984: 59)

C'est la seconde partie de la chanson (postresalica), que Fortis décrit comme une «longue exclamation en vibrato», qui représente le plus grand défi technique du chanteur. Il ne s'agit pas ici d'un vibrato naturel, mais d'un élément mélismatique stylisé et affiné qui entraîne généralement une ornementation complexe (mordant et tremolo), c'est-à-dire la rapide réitération de groupes de deux, trois, quatre tons ou même davantage, le plus souvent chromatiques. Une bonne articulation d'ornementations déployées en une longue série mélodique laisse une impression artistique durable: c'est là que réside la virtuosité mélodique. Les ornementations sont accentuées par des obturations glottales qui provoquent des vibrations supplémentaires des cordes vocales, dont la hauteur est alors maîtrisée (elles mettent en œuvre des tons de l'échelle traditionnellement observée); aussi des groupes d'ornementations vocales sont-ils répétés de manière semblable. Ce mode de production des chansons vibrantes exige des cordes vocales élastiques et une colonne d'air suffisante, qui assurent également l'intensité et la durée de la formule mélodique.

Les deux facteurs revêtent une importance à la fois stylistique et esthétique dans la tradition vocale des Alpes dinariques. Or certaines chansons individuelles, comme les chansons vibrantes tirées des épopées, requièrent des cellules mélismatiques plus brèves, auxquels s'ajoute une exlamation à la fin de chaque vers, afin que l'attention ne soit pas détournée de la substance du texte. Ce type de chant possède une dynamique moindre, parce que les chants épiques sont exécutés dans une maison, contrairement aux autres chansons vibrantes, et parce que le volume de la colonne d'air diminue beaucoup. Dans les chansons mentionnées, il faut unifier l'intensité et la durée de chaque vers pour en faire une unité mélodico-poétique.

Dans d'autres types de chansons vibrantes, comme les chansons des voyageurs, les chansons à boire et les turčije, l'élément mélismatique revêt plus d'importance, si bien que la formule entière s'en trouve allongée. C'est pourquoi le chanteur s'arrête entre la partie syllabique et la partie mélismatique. C'est une pause stylisée génératrice de tension: on attend la partie ornementale de la chanson.

Le terme de morlak (ou vlah) s'applique aux descendants des peuples autochtones des Balkans (Daces roumains, Dardaniens et Illyriens), de même qu'aux paysans et bergers d'origine chrétienne, établis en Yougoslavie centrale. 


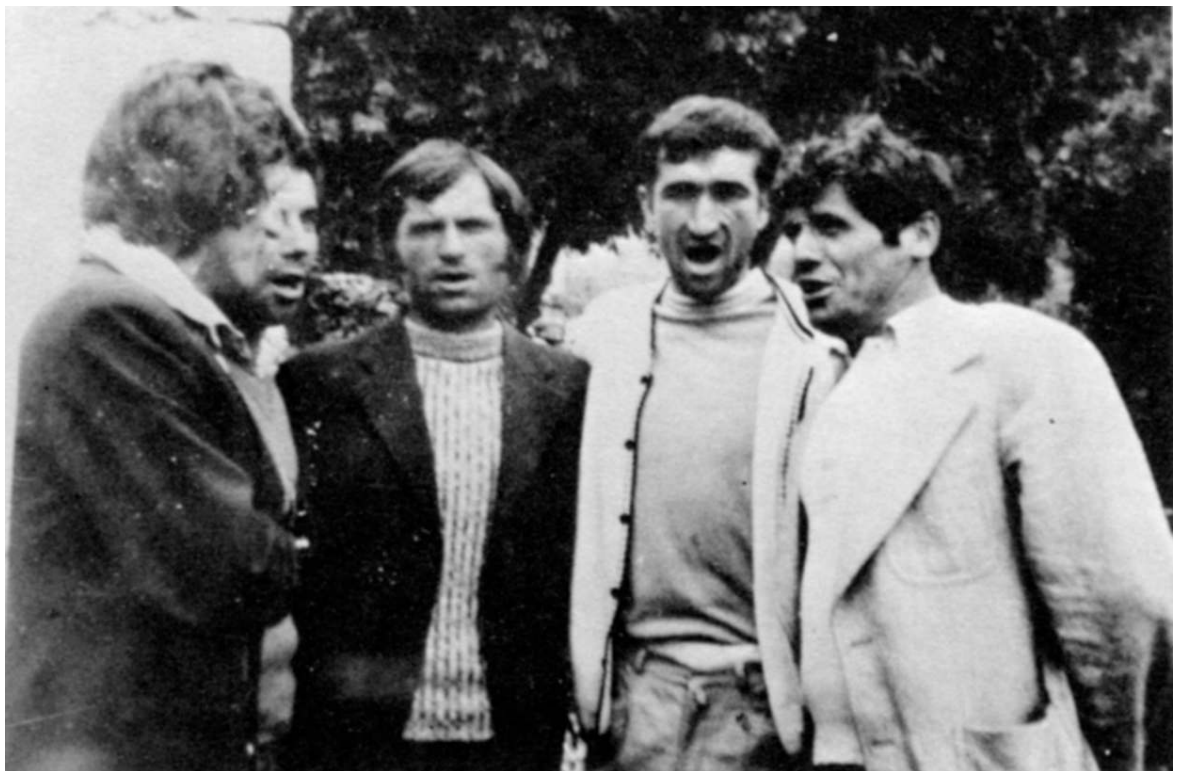

Fig. 7: Interprétation d'une ganga, Herzégovine orientale.

Dans les chants diaphoniques, où le soliste exécute d'abord la partie syllabique, la ligne mélodique revient à un chanteur à la voix reposée, alors que d'autres chanteurs exécutent la partie syllabique ou seulement le bourdon. Dans les chansons plus longues à deux voix, la formule mélodique passe d'une voix à l'autre, afin que la continuité mélodique du chant se maintienne aussi longtemps que possible. Il va de soi que dans ces cas, tous les chanteurs doivent être à même d'orner la mélodie. Ici la mobilité de l'organe vocal est presque égale à celle du diple.

La seconde catégorie de chansons, celle des $\operatorname{ganga}^{7}$, se retrouve dans toute l'Herzégovine et le Zagora dalmatien. Ce genre vocal se distingue par un type différent d'ornementation relativement brève, chantée sur les voyelles a, e, d ou o, ou bien, à Zagora, sur la syllabe re (ici la dénomination rera se substitue donc au terme ganga). C'est par ces ornementations que commence chaque note principale de la partie vocale d'«accompagnement» dans la section polyphonique de la chanson. Certains chanteurs confirmés utilisent des groupes de deux, trois ou quatre notes ornementales très brèves à la place d'un seule - ce qui exige, à côté d'une technique vocale exceptionnelle, la capacité de les composer sur-le-champ dans les limites du rythme établi par le meneur.

Le terme de ganga désigne la forme la plus répandue du chant polyphonique dans les Alpes dinariques; il dérive du terme albanais këngë, qui signifie «chant». 
A l'instar de toutes les autres chansons villageoises de Yougoslavie centrale, les ganga se caractérisent par une tessiture étroite située dans le registre moyen de la voix. Les ornementations initiales, désignées par le terme populaire de jecanje («sangloter») ou sjecanje («trancher»), sont exécutées dans le registre aigu comme des harmoniques. Elles sont gutturales, et ceux qui n'y sont pas habitués ont l'impression qu'elles accentuent les tons principaux.

On retrouve des ornementations semblables dans la musique traditionnelle iranienne, où elle sont appelées takiyah. Margaret Caton (1974: 43) décrit ce style comme «une ornementation en falsetto ou yodlée, un des éléments les plus caractéristiques et essentiels du style vocal iranien». Elle illustre son article par des graphiques du mélographe, fournissant ainsi une image sonore plus précise. Elle décrit le takiyah comme un style musical «qui vise à "sangloter" sur une note, le chanteur se concentrant plus sur l'emprise émotionnelle que sur telle ou telle note».

Ainsi, tant dans le takiyah iranien que dans la ganga, les ornementations précèdent les sons principaux et s'appellent «sanglots », à la différence près que les «sanglots» de la ganga se situent toujours à la même hauteur dans une seule et même exécution, contrairement au takiyah.

Les gens pensent qu'il faut avoir «une bonne gorge» pour être en mesure d'exécuter convenablement la partie sanglotante des ganga. Cela exige une technique adéquate de maîtrise des organes respiratoires. Chacun des éléments caractéristiques de la musique des montagnes dinariques est d'une importance égale, tout en étant valorisé du point de vue esthétique. Ceux qui répondent le mieux aux normes vocales établies sont les jeunes aux organes vocaux sains et élastiques.

Dans les ganga à signification collective, pour favoriser l'exécution et la disponibilité auditive, il faut bien choisir les chanteurs. Ainsi chaque village possède ses «bons chanteurs» de ganga, et ceux-là ne sont pas très nombreux.

L'apprentissage des ganga comme des autres chansons ne se fait pas en public mais en cachette, au moment de la puberté (c'est-à-dire lorsque la voix a atteint sa maturité), par l'imitiation des chanteurs confirmés. C'est seulement quand ils ont trouvé leur style et une exécution sûre et qu'ils méritent le respect de la communauté en tant qu'adultes, que les plus jeunes chanteurs osent se produire en public. Lorsque les interprètes confirmés commencent à avoir des difficultés, que le style, l'esthétique ou l'exécution technique en souffrent, des chanteurs plus jeunes prennent la relève. Ils doivent être capables de chanter à l'unisson et de coordonner l'application de toutes les autres normes stylistiques léguées par la tradition.

En conclusion, on peut affirmer que les techniques vocales décrites dans ces pages ne se ramènent pas seulement à l'activité des composantes de l'organe vocal humain, producteur de phénomènes musicaux. Au contraire, elles sont en elles-mêmes déjà un facteur du style musical, dont les déterminantes sont imposées et valorisées socialement, voire culturellement; et c'est en cela que le style de chant décrit ici diffère notablement d'autres styles vocaux. 


\section{Bibliographie}

\section{CATON Margaret}

1974 «The vocal ornament takiyah in Persian music». Selected Reports in Ethnomusicology II(1) : 42-53.

FORTIS Alberto

1984 Put po Dalmaciji. Zagreb: Globus.

HOOD Mantle

1971 The Ethnomusicologist. New York: McGraw Hill.

LOMAX Alan

1968 Folk Song Style and Culture. New Brunswick, N.J.: Transaction Books.

MUNISHI R.

1987 Këngët Malësorçe Shquiptare. Instituti Albanologijk i Prishtinës.

PETROVIĆ Ankica

1977 Ganga, a Form of Traditional Rural Singing in Yugoslavia. Belfast: The Queen's University [Unpublished $\mathrm{PhD}$ Thesis].

TAYLOR C.A.

1965 The Physics of Musical Sounds. New York: American Elsevier. 